\title{
Full Reference-Image Quality Assessment with Global and Local Image Regions for Visual Quality Perception and Optimization
}

\author{
Syed Shanawaz, G.Amjad Khan
}

\begin{abstract}
Image Quality Assessment (IQA) by using mathematical methods is offering favorable results in calculating visual quality of distorted images. These methods are developed by examining effective features that are compatible with characteristics of Human Visual System (HVS). But many of those methods are difficult to apply for optimization problems. This paper presents DCT based metric with easy implementation and having mathematical properties like differentiability, convexity and valid distance metricability to overcome the optimization problems. By using this method we are able to calculate the quality of image as a whole as well as the quality of local image regions.

Keywords:Human Visual System, Image Quality Assessment, Image quality optimization problem.
\end{abstract}

\section{INTRODUCTION}

IMAGE quality is a characteristic of the image that tells about how much amount of image is degraded due to different distortions like noise, blurring, fading. Image degradation occurs during image acquisition, transmission, compression, decompression, printing etc. The main aim of many image processing based applications is to improve the visual quality of the image or to reduce the distortions that are present in the image. In this regard lot of work has been going on for developing mathematical Image Quality Assessment (IQA) methods whose results should be compatible with perceived visual quality.

The quality of the image is obtained either by using subjective methods or by using objective methods. In subjective way of quality assessment human observers will rate the image quality. The distorted image is given to image processing experts and they will decide the quality of the image by using Mean Opinion Score (MOS), which is the subjective quality assessment method. In objective methods the image quality is obtained by using computations or algorithms.

Depending on the availability ofideal image or reference image information at the time of quality assessment the objective image quality metrics are classified into three types: i) Full-Reference IQA , ii) Reduced-Reference IQA and iii) No-Reference IQA. In Full-Reference IQA (FRIQA) the distorted image is compared with original or reference image for obtaining the quality of the image. In Reduced-Reference IQA (RR-IQA) only partial amount of original image is available at the time of assessment to evaluate the quality of degraded image. In No-Reference

Revised Manuscript Received on April 12, 2019.

Syed Shanawaz,M. Tech (CSP), Department of ECE, G. P. R. E. C, Kurnool, A.P, India.(Email: syedshanawaz5295@gmail.com)

G.Amjad Khan,Assistant Professor, Department of ECE, G. P. R. E. C, Kurnool, A.P, India. (Email: gak80rec@gmail.com)
IQA (NR-IQA) only the distorted image is available and original image is not available. This paper follows FR-IQA method which is illustrated in Fig.1.

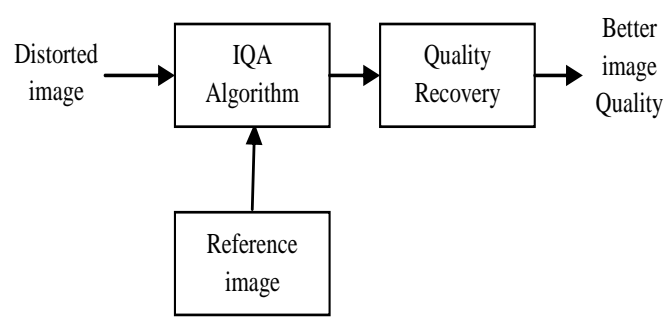

Fig. 1.Full-Reference Image Quality Assessment (FR-IQA)

The objective IQA methods should be compatible with subjective quality scores and characteristics of Human Visual System (HVS).

Contributions

i. In this paper we first discuss about the Human Visual System (HVS) and its characteristics based on different image texture characteristics and distortion types. We go through some important FR-IQA methods based on structural comparison.

ii. The proposed method is discussed later by using DCT as subband and by considering Additive Gaussian Noise (AGN) and Gaussian Blur (GB) distortions.

iii. The experimental results of different FRIQA methods are discussed in section IV.

\section{LITERATURE REVIEW}

\section{A. Characteristics of HVS}

The Visual system is the part of the central nervous system which gives organisms the ability to process visual detail. There are two important Human Visual System (HVS) characteristics:

i. Spatial Contrast Sensitivity function (CSF) and

ii. Contrast masking (CM) effect.

These characteristics have been applied in some previous FR-IQA methods.

According to spatial CSF, Human Visual System (HVS) has different sensitivities to distortions depending on spatial frequency in cycles per degree (cpd). Fig. 2 shows the example of spatial CSF. In this example, a sinusoidal grating pattern is introduced into the gray background and it shows

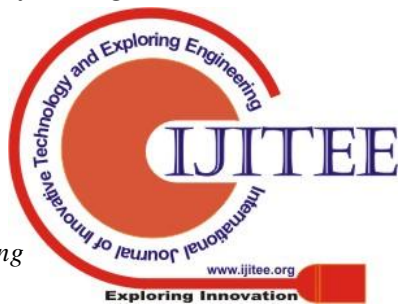


the relationship between the spatial frequency and contrast ratio. In Fig. 2, as the spatial frequency of the sinusoidal grating pattern increases along the $\mathrm{x}$-axis the contrast ratio of the sinusoidal grating pattern decreases along the y-axis. Fig. 2 shows how the changes in the sinusoidal grating pattern with respect to spatial frequency affect the visual perception of HVS, and it shows the band pass property.

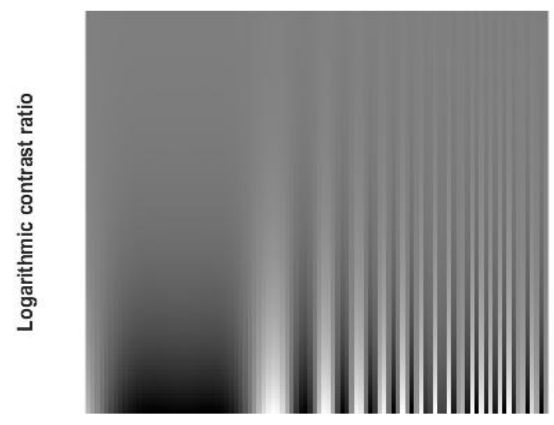

Logarithmic spatial frequency

Fig. 2.An example of spatial CSF

On the other hand, according to contrast masking (CM) effect [13], Human Visual system (HVS) has different sensitivities to distortions depending on background image texture characteristics. There are different image texture regions like homogeneous image texture region, complex image texture region and edge image texture region. HVS responds differently for different noises or distortions. For example, if the original image is distorted with Pseudo additive white Gaussian noise (AWGN) then it will become the distorted image given as $\mathrm{V}=\mathrm{U}+\mathrm{N}$, where $\mathrm{U}, \mathrm{N}$ and $\mathrm{V}$ represents the original image, AWGN distortion and distorted image respectively.

It becomes easy for Human Visual System (HVS) to find that the image is affected with AWGN distortions in homogeneous image texture region where as it becomes difficult to find AWGN distortions in complex image texture region. Similarly this is different for HVS when other distortions were considered. From this we can say that Human Visual System (HVS) perceives different visual quality to different distortions depending on image texture characteristics.

\section{B. Existing FR-IQA metrics}

This section deals with some important existing FR-IQA methods. These methods uses different models like similarity measure based models, normalized distance metric based models, internal brain mechanism based models.

One of the most widely used distortion metric is Mean Square Error (MSE) [1], which serves both finding image quality and it also optimizes image quality algorithms. Even though it is easy to compute and has valid mathematical properties like distance metricability, differentiability and convexity, the problem is its poor correlation with perceived subjective quality scores.

To overcome this problem many FRIQA methods [1-14] have been proposed. Structural Similarity Index (SSIM) is considered as the milestone work in FRIQA modeling problems. Some important structure based FRIQA methods include Multiscale Structural Similarity Index (MSSIM),
Riesz transform based Feature Similarity Index (RFSIM) [5], Feature Similarity Index (FSIM) [6], Gradient Similarity (GSM) based method [7], Gradient Magnitude Similarity Deviation (GMSD) [8], and Visual Saliency induced Index (VSI) [9].

Recently, Structural Contrast-Quality Index (SC-QI) and Structural Contrast-Distortion Metric (SC-DM) [10] have been proposed in which entire image is divided into $4 \times 4$ blocks called as local image regions. Combining all the local quality values gives the global quality i.e., the quality of entire image.

The local SCQI value is given as

$f(u, v)=\prod_{k=1}^{6} s_{k}$

where $u, v$ are the local images of the original image $U$ and distorted image $V$ respectively. $s_{k}, \quad k=1,2, \ldots 6$ represents the six similarity measures between the original and distorted images. The similarity is calculated between the features Structural Contrast Index (SCI), contrast measurement and chrominance measurement of both reference (or original) image and distorted image [10].

In SC-DM, instead of using similarity measure, distortion measure between reference and distorted images is considered. The local SC-DM value is calculated by using normalized mean square error (NMSE) and is given as

$$
\begin{aligned}
& f(u, v)=\|d\|_{2}^{2} \\
& \text { The global quality is calculated as } \\
& F(U, V)=E[f]=\frac{1}{W} \sum_{j=1}^{J} w\left(u^{j}, v^{j}\right) f\left(u^{j}, v^{j}\right)
\end{aligned}
$$

where $J$ in (3) represents the number of $4 \times 4$ local image blocks in $U$ and $V, w(u, v)$ is the local weight with respect to local importance and $W$ is equal to sum of all $w(\mathrm{x}, \mathrm{y})$ values over all $J$ local image blocks and acts as a scaling factor.

Even though SC-QI and SC-DM have given hopeful results, they sometimes fail precisely predict visual quality perception. Hence, they are mathematically hard to be able to manage in optimization problems. So, we present a FRIQA method by using features in [14] with easy implementation and to overcome optimization problems.

\section{PROPOSED METHOD}

Many FRIQA methods recently have been existed based on high-level assumptions such as extracting structural information from image textures for visual quality of Human Visual System (HVS) characteristics, whereas our proposed work is based on low-level assumption i.e., perceived image quality is associated with difference of neural responses between the reference and distorted image signals.

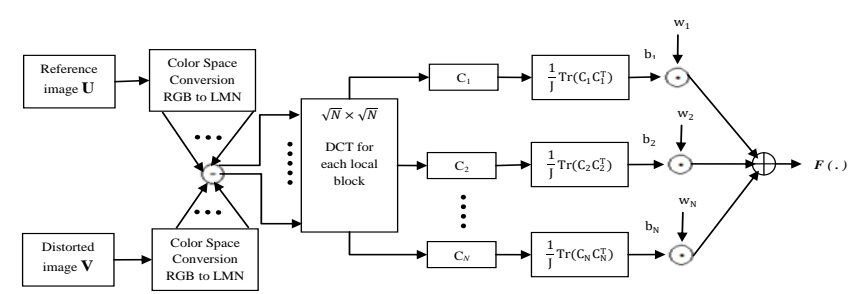

Fig. 3. Computation diagram of proposed method

Blue Eyes Intelligence Engineering 
To derive the quality of degraded image, we consider DCT domain as the subband that replicates neural responses in visual cortex. Our proposed method is derived based on probability summation theory and a psychometric function. We have to consider a psychometric function whose purpose is to serve as an individual detection probability model for distortion at $n$-th DCT coefficient, which is given as

$$
\left(P_{n}\left(d_{n}\right)\right)=1-\exp \left\{-\left(g_{n} \cdot d_{n}\right)^{p}\right\}
$$

where $d_{n}=\left|u_{n}-v_{n}\right|$ is the difference between the original and distorted coefficient values. $g_{n}$ is the measured sensitivity value for distortion and $p$ is a model parameter. The example of detection probabilities for quality degradation with respect to signal importance is shown in Fig. 4.

By extending (4) to each coefficient in a DCT block, the local image degradation value is given as

$$
f_{j}\left(u_{j}, v_{j}\right)=\sum_{n=1}^{N}\left(g_{n} \cdot d_{j, n}\right)^{p}
$$

The whole quality degradation value for the distorted image $V$ as compared to original image $U$ is defined by combining all the local degradation values and is given as

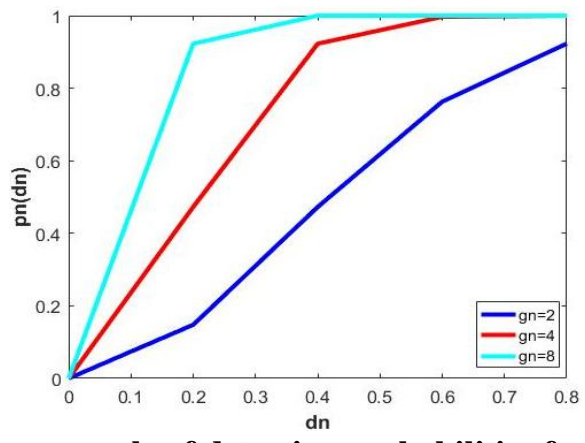

Fig. 4.An example of detection probabilities for quality degradation with respect to signal importance

$$
F(U, V)=\frac{1}{J} \sum_{j=1}^{J} \sum_{n=1}^{N}\left(g_{j, n} \cdot d_{j, n}\right)^{p}
$$

where $J$ is the total number of local DCT blocks. We set $p=2$ and assume $g_{j, n}$ to be locally independent (i.e., $g_{j, k}=g_{k}, j=1,2, \ldots J$ ) and is fixed for each subband in (6).

Our proposed method can be simply formulated as

$F(U, V)=B^{T} W$

$W=\left[w_{1}, w_{2}, \ldots . ., w_{N}\right]^{T}$, where $w_{n}=v_{n}^{2}, n=1,2, \ldots \ldots, N$ and $B=\left[b_{1}, b_{2}, \ldots \ldots, b_{N}\right]^{T}$ is obtained for each subband by using

$$
b_{n}=\frac{1}{J} \sum_{j=1}^{J}\left(u_{j, n}-v_{j, n}\right)^{2}
$$

The computation diagram of proposed method is shown in Fig. 3. $C_{n}$ is the coefficient map consists of $n$-th coefficients of all the $\sqrt{N} \times \sqrt{N}$ local DCT blocks.

The final form of proposed metric in (7) is as same as that of [14] and is very simple. In this method, the number of features $(N)$ is 48 which are obtained from three color channels $(\mathrm{LMN})$, each of the color channel uses $4 \times 4$ DCT block having 16 coefficients. In this, we use $N=16$ for each color channel. As in most existing FR-IQA methods, downsampling by a factor of 2 using a $2 \times 2$ uniform kernel is applied before computing local quality. This reduces the computational complexity and it rarely affects the performance degradation. The local quality values of the proposed method are calculated using a sliding window approach with every one pixel shift, which helps in detecting the blockiness artifacts in distorted images.

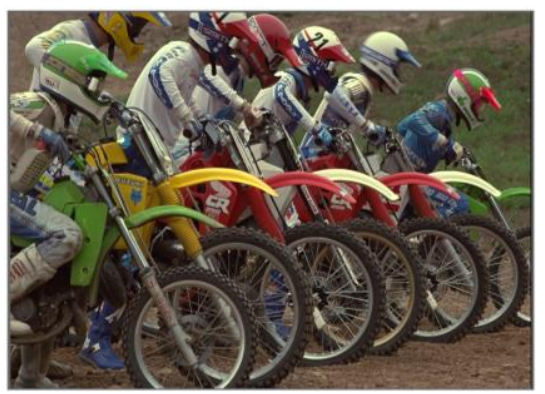

Fig. 5. Original image $U$

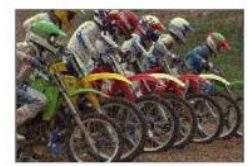

(a)

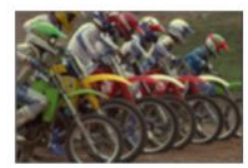

(d)

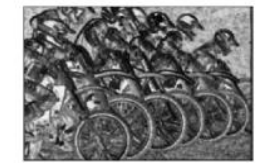

(b)

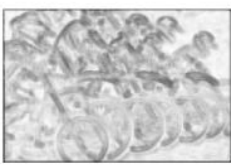

(e)

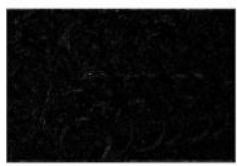

(c)

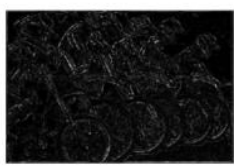

(f)
Fig. 6. Distorted images by additive Gaussian noise (AGN) and Gaussian blur (GB) from the original image $X$ in Fig. 6. SC-QI and SC-DM maps are computed in each row. (a) AGN distortion of $X$ denoted by $Y_{1}$ (b) SCQI map of $Y_{1}$. (c) SC-DM map of $Y_{1}$ (d) GB distortion of $X$ denoted by $Y_{2}$.(e) SC-QI map of $Y_{2}$. (f) SC-DM map of $\mathbf{Y}_{2}$.

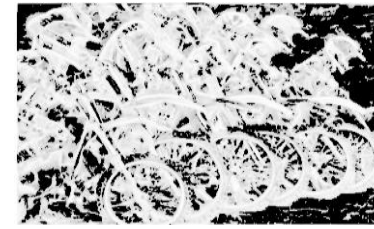

(a)

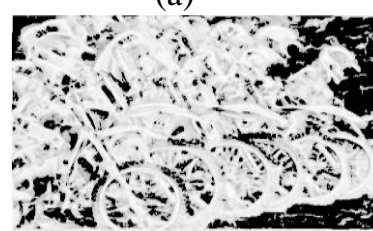

(c)

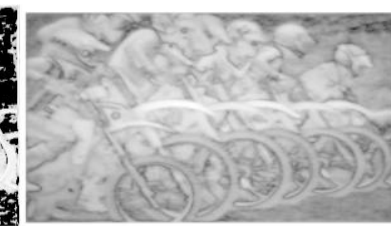

(b)

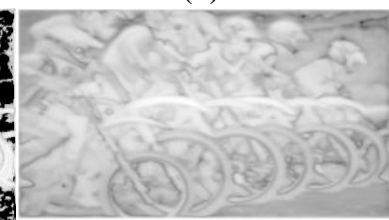

(d)
Fig. 7.Mapping of FSIM $_{C}$ and VSI for GB distortions (first row) and AGN distortions (second row). (a) FSIM $_{C}$ map of $Y_{1}$ (b) VSI map of $Y_{1}$ (c) FSIM $_{C}$ map of $Y_{2} \cdot$ (d) VSI map of $Y_{2}$.

\section{EXPERIMENTAL RESULTS}

As discussed earlier Human Visual System (HVS) perceives visual quality depending on different image texture characteristics and various distortion types. In this method we use two types of distortions; pseudo Additive Gaussian Noise (AGN) and pseudo Gaussian Blur (GB).

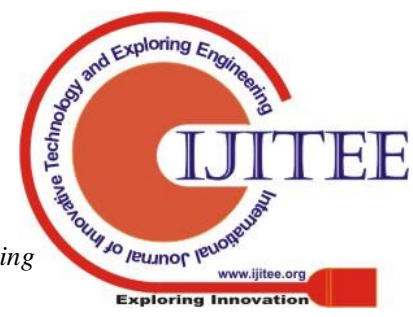


Fig. 5 shows the original (or reference) image $U$ with size $512 \times 384$ which is used to analyze the behavior of some important FR-IQA methods including SC-QI and SC-DM [10]. The response of SC-QI and SC-DM due to these two distortions is also shown in Fig. 6.

In this paper, we not only concentrated on proposed metric and SC-QI and SC-DM, but also analyzed the performance of other important FR-IQA methods. Fig. 7 shows the mapping of local image regions of VSI and FSIM $_{C}$ for both additive Gaussian noise (AGN) and Gaussian blur (GB) distortions. In Fig. 7, the first column denotes the local maps of Feature Similarity $\left(\right.$ FSIM $\left._{C}\right)$ [6] and second column represents the local quality maps of Visual Saliency induced Index (VSI) [9] respectively. Similarly, the first row in Fig. 7 denotes the local map of FSIM $_{\mathrm{C}}$ and VSI due to pseudo Gaussian blur (GB) distortions and second row denotes the local maps of FSIM $_{C}$ and VSI due to additive Gaussian noise (AGN) distortions. In our experiments, total eight FR-IQA methods are compared, i.e., SSIM [2], GSM [7], M-SSIM [3], FSIM [6], RFSIM [5], VSI [9], and SC-QI (SC-DM) [10] are compared with the proposed quality metric in terms of finding the quality of distorted images.

\section{TABLE I PERFORMANCE COMPARISON OF DIFFERENT FR-IQA METHODS}

\begin{tabular}{ccc}
\hline \hline FR-IQA method & AGN Distortions & GB Distortions \\
\hline SSIM & 0.6209 & 0.7792 \\
MSSIM & 0.8918 & 0.9255 \\
RFSIM & 0.5811 & 0.8986 \\
FSIM & 0.9412 & 0.9039 \\
GSM & 0.9708 & 0.9558 \\
VSI & 0.9722 & 0.9650 \\
SCQI & 0.9727 & 0.9718 \\
SCDM & 0.9728 & 0.9718 \\
Proposed metric & $\mathbf{0 . 9 7 3 5}$ & $\mathbf{0 . 9 7 4 1}$ \\
\hline \hline
\end{tabular}

Table I shows the quality values obtained for both additive Gaussian noise(AGN) distortions and pseudo Gaussian blur (GB) distortions for the above mentioned FRIQA methods. From the performed experiments, it is obvious that the proposed method is easy to implement and it provides valid results for quality measures.

It is shown in Table I that each method has different performance to different distortions. The proposed metric produces promising results to different distortions for image quality optimization problems. This indicates that the proposed method can be effectively applied in image compression problems with respect to optimizing perceptual visual quality.

Different color spaces will show effect on the behavior of classification accuracies for some image classification and recognition tasks. The color space conversion used in other image quality metrics [6], [14] is RGB to YIQ. We compare the performance of DCT-based quality metric for YIQ color space with LMN color space.

\section{TABLE II PERFORMANCE COMPARISON OF PROPOSED DCT-BASED METRIC FOR LMN AND YIQ COLOR SPACES}

\begin{tabular}{ccc}
\hline \hline Color space & AGN Distortions & GB Distortions \\
\hline LMN & 0.9735 & 0.9741 \\
YIQ & 0.9707 & 0.9721 \\
\hline \hline
\end{tabular}

Table II shows the performance of DCT-based quality metric for LMN and YIQ color spaces. It is noticed that YIQ color space has comparable results against LMN color space, indicating that proposed metric can effectively be applied for different applications with their dedicated color spaces. These experiments are performed on MATLAB 9.0 (R2016a) software.

\section{CONCLUSION}

In this paper, a FR-IQA method is presented by considering that HVS has different strategies in perceiving visual quality. The proposed metric has mathematically desirable properties of differentiability, convexity and valid distance metricability and is of less computational complexity as compared to other metrics. The experimental results show that proposed metric produces better results as compared to other metrics. This can be effectively used for image quality optimization problems with known distortion types. With some modification the proposed metric can also be used with different DCT kernel sizes for improving the perceptual visual quality.

\section{REFERENCES}

1. Z. Wang and A. C. Bovik, "Mean squared error: Love it or leave it? A new look at signal fidelity measures," IEEE Signal Process. Mag., vol. 26, no. 1, pp. 98-117, Jan. 2009.

2. Z. Wang, A. C. Bovik, H. R. Sheikh, and E. P. Simoncelli, "Image quality assessment: From error visibility to structural similarity," IEEETrans. Image Process., vol. 13, no. 4, pp. 600-612, Apr. 2004.

3. Z. Wang, E. P. Simoncelli, and A. C. Bovik, "Multiscale structural similarity for image quality assessment," in Proc. Conf. Rec. 37 ${ }^{\text {th }}$ Asilomar Conf. Signals, Syst., Comput., Nov. 2003, pp. 1398-1402.

4. Z. Wang and Q. Li, "Information content weighting for perceptual image quality assessment," IEEE Trans. Image Process., vol. 20, no. 5. pp. 1185-1198, May 2011.

5. L. Zhang, D. Zhang, and X. Mou, "RFSIM: A feature based image quality assessment metric using Riesz transforms," in Proc. 17th IEEE

6. Int. Conf. Image Process., Sep. 2010, pp. 321-324.

7. L. Zhang, L. Zhang, X. Mou, and D. Zhang, "FSIM: A feature similarity index for image quality assessment," IEEE Trans. Image Process., vol. 20, no. 8, pp. 2378-2386, Aug. 2011.

8. A. Liu, W. Lin, and M. Narwaria, "Image quality assessment based on gradient similarity," IEEE Trans. Image Process., vol. 21, no. 4, pp. 1500-1512, Apr. 2012.

9. W. Xue, L. Zhang, X. Mou, and A. C. Bovik, "Gradient magnitude similarity deviation: A highly efficient perceptual image quality index," IEEE Trans. Image Process., vol. 23, no. 2, pp. 684-695, Feb. 2014.

10. L. Zhang, Y. Shen, and H. Li, "VSI: A visual saliencyinduced index for perceptual image quality assessment," IEEE Trans. Image Process., vol. 23, no. 10, pp. 4270-4281, Oct. 2014.

11. S.-H. Bae and M. Kim, "A novel image quality assessment with globallyand locally consilient visual quality perception," IEEE Trans. ImageProcess., vol. 25, no. 
12. D. Brunet, E. R. Vrscay, and Z. Wang, "On the mathematical properties of the structural similarity index," IEEE Trans. Image Process., vol. 21, no. 4, pp. 1488-1499, Apr. 2012.

13. J. Wu, W. Lin, G. Shi, and A. Liu, "Perceptual quality metric with internal generative mechanism," IEEE Trans. Image Process., vol. 22, no. 1, pp. 43-54, Jan. 2013.

14. G. E. Legge and J. M. Foley, "Contrast masking in human vision," J. Opt. Soc. Amer., vol. 70, no. 12, pp. 1458-1471, Dec. 1980.

15. S.-H. Bae and M. Kim, "DCT-QM: A DCT-based quality degradation metric for image quality optimization problems," IEEE Trans. ImageProcess., vol. 25, no. 10, pp. 4916-4930, Oct. 2016. 